Umwelt und Entwicklung, Rio : Fünf Jahre danach

\title{
Zusammenarbeit zwischen Madagaskar und der Schweiz : die Umweltdimension
}

Michèle Rajaonarivony

\section{OpenEdition}

1 Journals

Electronic version

URL: http://journals.openedition.org/sjep/757

DOI: 10.4000/sjep.757

ISSN: 1663-9677

Publisher

Institut de hautes études internationales et du développement

Printed version

Date of publication: 1 mars 1997

Number of pages: 261-276

ISSN: 1660-5926

\section{Electronic reference}

Michèle Rajaonarivony, « Zusammenarbeit zwischen Madagaskar und der Schweiz : die

Umweltdimension », Schweizerisches Jahrbuch für Entwicklungspolitik [Online], 16 | 1997, Online erschienen am: 14 August 2012, abgerufen am 08 September 2020. URL : http://

journals.openedition.org/sjep/757 ; DOI : https://doi.org/10.4000/sjep.757 


\title{
ZUSAMMENARBEIT ZWISCHEN MADAGASKAR UND DER SCHWEIZ : DIE UMWELTDIMENSION*
}

\author{
Michèle Rajaonarivony
}

V

or dem Hintergrund des weltweiten Wettrennens um Wachstumsraten und der Bevölkerungsexplosion ist der Eintritt in das dritte Jahrtausend für die am wenigsten entwickelten Länder mit Schwierigkeiten verbunden. Die Umweltzerstörung, ein Resultat des Raubbaus an natürlichen Ressourcen, und die Verarmung der Bevölkerung dieser Länder sind für die Entwicklungsorganisationen zu einem wesentlichen Anliegen geworden. Sie sehen ihre Aufgabe darin, die Partner im schwierigen Lernprozess, der eine nachhaltigere Nutzung ihrer natürlichen Ressourcen erlauben soll, zu begleiten. Aus dieser Perspektive bemühte sich die Direktion für Entwicklung und Zusammenarbeit (DEZA), die Umweltdimension im Programm über die Zusammenarbeit mit Madagaskar stärker zu betonen. Nach einer Einführung in das madagassische Umfeld und einer Schilderung der spezifischen Umweltproblematik der Grossen Insel möchten wir die verschiedenen Ansätze des von der DEZA unterstützten Zusammenarbeitsprogrammes erklären. Der Schwerpunkt liegt dabei auf den Herausforderungen und den gewonnenen Erkenntnissen. Mit der Diskussion der Erfolge und Misserfolge ziehen wir danach die Bilanz aus dem Zusammenarbeitslansatz, der die Umwelt - den Lebensraum der gesamten Bevölkerung - in den Mittelpunkt stellt.

\section{EIN TAG AUF DEM MADAGASSISCHEN HOCHLAND}

An einem schönen Sonntagnachmittag bereitet sich das kleine Dorf, etwa fünfzehn Häuser, auf das Fest vor. Ein leichter Wind streift die roten Backsteinfassaden der Häuser, denen über hundert Jahre alte Feigenbäume Schatten spenden. Hier und da steigen noch Rauchsäulen von den typischen Strohdächern empor. Sobald die ersten Trommelschläge ertönen, verlassen die Bewohner ihre Häuser und strömen zum Treffpunkt. Auf dem Feld, wo normalerweise Fussball gespielt wird, ist bereits eine dichtgedrängte Menschenmenge zu sehen. Viele Familien kauern oder sitzen im Schneidersitz auf dem trockenen, kurzgeschnittenen Gras. Die mit Strohhüten oder Tüchern bedeckten Köpfe bewegen sich in alle Richtungen ; man hält nach Nachzüglern Ausschau oder bewundert die Künstler. Kinder und Erwachsene machen Kommentare zu den Aufwärmübungen des jüngsten Tänzers der Gruppe. Sobald die mpihira gasy spüren, dass Ausdruck, Auftreten und Aufmerksamkeit optimal sind, stampfen sie im Takt der grossen Trommel mit den Füssen und klatschen zum Auftakt der Vorstellung in die Hände. Und nun setzt die Musik ein...

\footnotetext{
* Mein herzlicher Dank gilt Kuno Schläfli (Projektbeauftragter für Madagaskar in der DEZA-Zentrale von 1993 bis 1996) und Philippe Zahner (Koordinator der DEZA in Madagaskar von 1991 bis 1996) für ihre Erklärungen zur Fachliteratur und für ihre Antworten auf meine zahlreichen Fragen. Auch für die Überarbeitung meines Textes möchte ich ihnen bestens danken.
} 
Die mpihira gasy sind für Madagaskar, was die Troubadoure einstmals für Europa waren. Sie reisen durch das Land und tragen traditionelle Lieder vor, deren Texte sie der Neuzeit anpassen. Die Vorstellung erfolgt als Sprechgesang zwischen zwei Seiten, Männern und Frauen ; die einen kritisieren, die andern kontern usw. Es geht jeweils darum, Missstände zu verurteilen bzw. sie zu rechtfertigėn. In bunte, fliessende Gewänder gekleidet, deklamieren die Tänzer Szenen aus dem Alltag und übermitteln wegweisende Botschaften auf humorvolle Weise. Mit ihren flinken blossen Füssen vollführen sie akrobatische Kunststiucke. Manche tanzen, andere singen - ein faszinierendes, populäres Schauspiel.

Die Neuheit des Tages : Unseren mpihira gasy geht es diesmal nicht um Korruption oder um Eifersucht, sondern um sehr viel alltagsnähere Themen. Die Liedertexte muten wie eine Litanei an : « Die Bäume rund um das Dorf ; die Felder, welche die Bauern pflügen ; die Hügel, auf denen ihr Vieh weidet ; das Wasser, das sie schöpfen - die Umwelt und die Landbewohner selbst sind in Gefahr ». Zum ersten Mal erfahren die Bauern, dass sie sich weniger verschulden können, wenn sie ihre Kulturen diversifizieren, oder dass sie Wasser sparen können, indem sie das Bewässerungssystem verbessern. Aus Aufmerksamkeit wird echtes, wenn auch mit einer gewissen Skepsis gemischtes Interesse. Eigentlich hätten die Bauern lieber konkrete Lösungsvorschläge für ihre Umweltprobleme gehört als Lieder. Denn Gepflogenheiten über den Haufen zu werfen, die sich seit Generationen bewährt haben, will gut überlegt sein ; an ihrer Stelle andere Praktiken einzuführen, fällt noch schwerer. Wenn dann die Warnungen zudem von Sängern stammen, die Bauern sind wie sie, kennt die Verwunderung keine Grenzen... Bei allem Staunen ist den Bauern aufgefallen, dass einige städtisch gekleidete Personen die Sänger begleiten - sicher nicht als Statisten : Geld, Material, Technik müssen also auf dem Spiel stehen. "Aber warum die mpihira gasy? "

Die schweizerische Entwicklungszusammenarbeit und der WWF unterstützen diese Künstler, weil sie es schaffen, auch die Herzen der Bauern zu erreichen. Genau daran sind viele Techniker und Agrarberater gescheitert. Mit einfachen, direkten und für alle verständlichen Worten erklären die Musiker die Zusammenhänge zwischen zu grossen Familien und Gefährdung der Wälder, unfruchtbaren Böden und Verschwinden heimischer Arten. Mit vorwurfsfreien, sachlichen Worten beschreiben sie die Lebensumstände der Bauern und weisen sie auf ihre eigene Verantwortung hin. Mit den Folkloretänzern übermitteln die schweizerische Entwicklungszusammenarbeit und der WWF die Umweltbotschaft über den kulturellen Kanal und erreichen die madagassische Bevölkerung an der empfänglichsten Stelle : der Freude an Wortgefechten und an der gesprochenen Sprache, der Macht der traditionellen Reden (kabary).

Die ländliche Entwicklung über die kulturelle Dimension anzugehen - zum Beispiel mit den mpihira gasy - ist nur einer von sechs Ansätzen, welchen Madagaskar und die Schweiz im Zusammenarbeitsprogramm für 1995-2000 zur Behandlung der Umweltproblematik anwenden. Die Palette der sechs verschiedenen Ansätze bildet das Ergebnis eines über zwanzig Jahre langen Lernprozesses, namentlich im Bereich der ländlichen Entwicklung, und fügt sich in den Kontext der Entschliessungen des Erdgipfels von 1992 in Rio de Janeiro ein. Bevor wir aber die madagassisch-schweizerischen Ansätze und vor allem die erzielten Resultate im einzelnen erörtern, versuchen wir, unsere Zuschauer beim mpihira gasy-Schauspiel in ihr Gesamtumfeld zu stellen : Madagaskar und seine Besonderheiten. 


\section{DIE UMWELT AUF MADAGASKAR : WAS STEHT AUF DEM SPIEL ?}

Die «grüne Revolution» kam nicht bis Madagaskar. Im übrigen ist dieser Begriff überholt ; längst sind die Konzepte "nachhaltige Entwicklung » und " gerechte Aufteilung der Wachstumsgewinne » in aller Munde. Die Grosse Insel verfügt über ein reiches, weltweit anerkanntes Potential. Madagaskar ist vierzehn Mal so gross wie die Schweiz (oder wie Frankreich und die Benelux-Staaten zusammengenommen) und beherbergt eine zu $85 \%$ endemische Flora und Fauna. Zahlreiche Reptilien, Fische, Orchideen, die meisten Lemuren und auch die meisten Arten des Baobabs sind nur hier zu finden. Neben seinem Naturreichtum besitzt Madagaskar eine Reihe von bereits erprobten oder potentiellen Medizinalpflanzen, einzigartige geologische und marine Formationen und eine noch deutlich traditionsgeprägte kulturelle Vielfalt.

Geologische Merkmale des madagassischen Hochlands sind die Laterit-Böden mit einer im allgemeinen spärlichen Vegetation ; Ausnahmen bilden die Vulkanböden im Westen und im Süden der Insel. Die durch die Erosion abgetragenen und von lavaka (durch Rieselwasser gebildete Bodeneinbrüche) durchzogenen, mit spärlichen Eukalyptusbäume und Pinien bewachsenen Hügel (tanety) fallen regelmässig Buschbränden zum Opfer. Trotzdem deckt das Hochland den Grundbedarf an Obst und Lebensmitteln der Hauptstadt und anderer Grossstädte bis zur Ostküste. Das übrige Landesgebiet an den Hängen zur Küstenebene hin besteht aus Sandstein-, Fels- oder Kalksteinsedimenten. Auf diesem wenig fruchtbaren Land befinden sich alle $\mathrm{z}$ wölf Naturschutzgebiete der Insel (mit einer Gesamtfläche von fast 600 '000 Hektar), einer der beiden Nationalparks sowie die Mehrzahl der rund zwanzig besonderen Schutzzonen.

\section{Madagaskar in Kürze ${ }^{1}$}

Bevölkerung : 13,4 Millionen Einwohner 1992 (Jahr der letzten Volkszählung)

Rund 25\% der Bevölkerung lebt in der Stadt

Bevölkerungswachstum 3,2\% pro Jahr

$81 \%$ der Bevölkerung arbeitet in der Landwirtschaft

Lebenserwartung bei der Geburt : 56,5 Jahre

1 Arzt pro 8'333 Personen

Gesundheitsausgaben : $1,3 \%$ des BIP

Ausgaben für die Grundausbildung : $2,3 \%$ des BIP

Fläche : 582'000 km2, davon 5,3\% Ackerboden, 26,6\% Wald. Abholzung : 1,2\% pro Jahr

BSP : 2,8 Milliarden US\$:

Pro Kopf-Einkommen : 230 US\$

Die Agrarproduktion stellt 33\% des BIP

Industrieproduktion : $14 \%$ des BIP

Gesamte Auslandschulden : 4,4 Milliarden US\$

Schuldendienst : $19 \%$ der Exporte

1 Regierung von Madagaskar, Ministère chargé du Plan, 1992, 1993, 1994. 
Exporte im Verhältnis zu Importen : 63\%

Internationale öffentliche Hilfe : rund 350 Millionen US\$1994, davon 29\% Produktionssektor, $45 \%$ Infrastrukturen, $18 \%$ Sozialsektor, Übriges Verwaltungssektor

Wichtigste Finanzquellen : IDA (35\%), Frankreich (24\%), Europäische Union (15\%), USA $(6 \%)$, Schweiz $(4 \%)$ und andere $(16 \%)$, darunter Deutschland, Japan usw.

Drei Viertel der Bevölkerung Madagaskars leben auf dem Land. Die Wirtschaft beruht im wesentlichen auf der Nutzung der natürlichen Ressourcen. Diese Ressourcen können sich jedoch nicht so rasch erneuern, wie die Bevölkerung wächst (jährlich um fast 3\%), und sind daher ernsthaft von Zerstörung bedroht. Nur noch 27\% der Fläche ist bewaldet. Jedes Jahr verschwinden weitere 200'000 Hektar Wald von der Landkarte. Anfang der 90er Jahre wurde gerade ein Zwanzigstel der Landesfläche systematisch für die Landwirtschaft genutzt ; lediglich ein Drittel davon kann bewässert werden. Dies verschärft die Armut der madagassischen Bevölkerung. Die Mehrheit - vier von fünf Menschen in der Stadt und drei von fünf auf dem Land - lebt derzeit unter der Armutsschwelle.

Die Lage ist alarmierend. Ein kürzlich erschienener Bericht ${ }^{2}$ des WEF-Sekretariats (World Environment Fund) anerkennt die weltweit einzigartige Artenvielfalt Madagaskars und erklärt, dass diese wegen ihres hohen Endemismus und angesichts der ernsthaften Bedrohung prioritären Schutz verdient.

Unter diesen Umständen hat sich die internationale Staatengemeinschaft auf einen Appell der Umweltbewegung massiv dafür eingesetzt, umfassende Projekte zum Schutz der einzigartigen Naturschätze des Landes zu finanzieren. Ziel ist die Unterstützung des nationalen «Plan d'Action Environnemental » PAE (Nationaler Umweltaktionsplan) vom Jahr 1992.

\section{Nationaler Umweltaktionsplan ${ }^{3}$}

Beginn : 1989

Grundlage : Umweltcharta

Dauer : 15 Jahre

Ausführung : in Phasen von 5 Jahren

Unterstützung : ein Geldgeber-Pool unter Federführung der Weltbank :

- Weltbank, UNDP, WEF und FAO

- Deutschland, Vereinigte Staaten, Frankreich, Japan, Norwegen, Schweiz und Europäische Union

Erste Phase : 1989 - 1995 (>1996)

- Schaffung eines rechtlichen Rahmens

- Ausarbeitung von Politiken und Strategien

- Errichtung der Ausführungsstrukturen

Zweite Phase : 1996 - 2001

- Konsolidierung der rechtlichen Arbeiten

- Konsolidierung der neuen Strukturen

${ }_{3}^{2}$ Global Environment Facility Proposal for Supporting Madagascar Environment Programme, unpubl., 1996.

${ }^{3}$ Regierung Madagaskar Charte de l'Environnement, 1990, 84 S. 
- Übergang von Pilotaktionen zu breitflächigeren operationellen Umsetzungen

- Neubestimmung der Prioritäten

Dritte Phase (geplant für 2001 bis 2006)

- Fortsetzung der breitflächigen operationellen Tätigkeiten

- Einbindung der Umweltstrategien in die sektoriellen Politiken und in die makroökonomische Politik des Landes

- «phasing out » (allmählicher Abbau) der institutionellen Unterstützung

Schwerpunkt in der zweiten Phase :

- nachhaltige Nutzung der Ressourcen an Boden und Wasser

- multifunktionelle Forst-Ökosysteme

- Bewirtschaftung geschützter Gebiete, Ökotourismus

- Meeres- und Küstenlandschaft

Die Umsetzung erfordert ein Budget von rund 30 Millionen US\$ jährlich, davon 7 Millionen US\$ von der madagassischen Regierung.

Der für eine Dauer von 15 Jahren konzipierte Aktionsplan wird in drei Projektphasen von jeweils 5 Jahren umgesetzt. Unterstützt wird der Plan hauptsächlich von der Entwicklungszusammenarbeit in den USA, Frankreich, Deutschland, der Schweiz und Japan, ferner von der Weltbank, dem WEF und den Organen der Vereinten Nationen.

\section{WELCHE ROLLE SPIELT DIE MADAGASSISCH-SCHWEIZERISCHE ZUSAMMENARBEIT ?}

Die Schweiz unterstützt zwar die Aktivitäten der internationalen Staatengemeinschaft, hält sie aber nicht für ausreichend, weil sie stärker auf die Erhaltung als auf die nachhaltige Nutzung der natürlichen Ressourcen im Interesse der Bevölkerung ausgerichtet sind. Zahlreiche nationale und internationale Partner tendieren dazu, die Umwelt auf Naturschutzgebiete, Nationalparks oder sogar auf bestimmte Waldbestände und Korallenriffe zu beschränken. Die DEZA definiert die Umwelt viel breiter, nämlich als Lebensraum der Bevölkerung mit ihren natürlichen Ressourcen und ihrer sozioökonomischen Struktur. ${ }^{4}$ Daher stellt die Beteiligung am PAE für die DEZA nur eine von sechs Ansätzen ihres Programmes dar.

4 Direktion für Entwicklung und Zusammenarbeit (DEZA), Umweltstrategie, 1994, $11 \mathrm{~S}$. 


\section{Zusammenarbeitsprogramm Schweiz-Madagaskar ${ }^{5}$}

Erste Schritte in den 60er Jahren mit einem Freiwilligenprogramm

Ende der 60er und Anfang der 70er Jahre : Aktivitäten in der Landwirtschaft, Forstwirtschaft und in der technischen Berufsbildung

Ende der 70er Jahre : Erweiterung auf die Universitätsausbildung (Viehzucht, Forstwirtschaft) und auf die Kleinindustrie

1977 wird ein Koordinierungsbüro errichtet

Anfang der 80er Jahre : Unterstützung des Gesundheitssektors (Basismedikamente, Trinkwasserleitungen) und der Strassentransportwege

Ende der 80er Jahre : Die Sorge um die Umwelt findet in der Beteiligung am Umweltaktionsplan einen konkreten Ausdruck

Anfang der 90er Jahre : Konzentration auf die folgenden Interventionsgebiete :

- nachhaltige und umweltverträgliche Produktion (Programme für die ländliche Entwicklung in drei ausgewählten Regionen, institutionelle Unterstützung bei der nachhaltigen Nutzung natürlicher Ressourcen, Unterstützung bei der Entwicklung sektorieller Politiken, Mitwirkung an Phase I und II des PAE)

- Aktionen im Sozialbereich (gemeinschaftlich organisierte Medikar.ıntenversorgung, Basismedikamente, Trinkwasserversorgung der Dörfer und andere gemeinschaftlich organisierte Projekte, Kommunikation im ländlichen Bereich, kulturelle Dimension)

- IStrassentransportwege (Reparatur und Unterhalt der wirtschaftlich wichtigen Strassenverkehrsachsen, Beteiligung des Privatsektors am Unterhalt der landwirtschaftlichen Zufahrtswege)

- Humanitäre Zusammenarbeit (Beteiligung an Interventionen der internationalen Staatengemeinschaft nach Zyklonen, Aktionen von « Vivre Contre Travail » zur Instandsetzung eines ausgedehnten Bewässerungsnetzes für die Landwirtschaft)

- Finanzielle Zusammenarbeit mit dem BAWI (Zahlungsbilanzhilfe im Gesundheitssektor, Reform des Finanzsektors)

In der zweiten Hälfte der 80er Jahre betrug das Budget der Bundesverwaltung für dieses Programm, einschliesslich der humanitären und finanziellen Zusammenarbeit, 20 bis 25 Millionen CHF ; heute sind es 17 bis 19 Millionen CHF.

Die Interventionen der Schweiz begannen vor rund dreissig Jahren im Bereich der technischen Bildung. Sehr rasch stellte sich die ländliche Entwicklung als wichtiger Punkt der madagassisch-schweizerischen Entwicklungszusammenarbeit heraus. Zudem ist es die eigentliche Aufgabe der DEZA, die Lebensbedingungen der ärmsten Bevölkerungsschichten und vor allem der Landbevölkerung, wenn sie die Mehrheit bildet, zu verbessern. Aus diesem Grund spielt die Sorge um die Umwelt auf allen Interventionsebenen mit.

Wenn nun die Karavanen der Schweizer ${ }^{6}$ und der madagassischen Musiker einen Monat lang durch die Insel ziehen und in ihren Liedern über Kultur und Umwelt sprechen; wenn Hunderte Hektar Sekundärwald im Westen der Insel verschont bleiben und die Bauern das brachgelegte Land nachhaltig nutzen können ; wenn

5 Auf der Grundlage von : DEZA, Länderprogramm Madagaskar 1995-2000, unpubl., 1994, 21 S. DEZA, Zusammenarbeitsprogramm Madagaskar-Schweiz 1994, 1995, 1996.

6 Die Berner Rockgruppe Patent Ochsner und der Waadtländer Akkordeonspieler Michel Besson. 
die kahlen und abgebrannten Hügel der Hochebene wieder bewirtschaftet werden ; kurz, ob die Projekte « Gasikara Maitso Volo » (Madagaskar, grüne Landschaften), « Action Monka ( (urbares Land) oder «Lova Soa » (wertvolles Erbe) heissen, die Beziehung zwischen der Landbevölkerung und ihrer Umwelt bildet bei allen Aktionen die wesentliche Herausforderung.

\section{WIE KÖNNEN MADAGASKAR UND DIE SCHWEIZ DIE HERAUSFORDERUNG BEWÄLTIGEN?}

Als Unterstützung der wirtschaftlichen Erholung des Landes erwarten die Madagassen von der Völkergemeinschaft einen Beitrag zur Vermittlung von Kompetenzen an ihre Staatsangehörigen und zum Aufbau des institutionellen Rahmens, den die aktive und wirksame Beteiligung am öffentlichen Leben voraussetzt. Jede in multilateraler oder bilateraler Zusammenarbeit durchgeführte Aktion soll der madagassischen Bevölkerung grössere wirtschaftliche und organisatorische Autonomie verschaffen.

Dieser Linie schliesst sich die schweizerische Entwickungszusammenarbeit eindeutig an, wobei die nachhaltige (umweltverträgliche) Entwicklung den Träger ihrer Intervention darstellt. ${ }^{7}$ Diese Wahl beruht auf drei Gründen. Einerseits gehört die Umwelt zu den fünf Prioritäten des Leitbildes ${ }^{8}$ der DEZA : Die «Unterstützung bei der Bewältigung von Umweltproblemen » ist ebenso wichtig wie das Erreichen einer «wirtschaftlichen Eigenständigkeit », die « Unterstützung der Armen », die « Verbesserung der Produktionsbedingungen » und die «Gesundheit und Bildung ». Andererseits konzentriert die schweizerische Entwicklungszusammenarbeit ihre Bemühungen in der Regel auf Bereiche, in welchen sie zu einem bestimmten nationalen Umfeld über einen reichen Ideenund Erfahrungsschatz verfügt. Dies trifft für die ländliche Entwicklung in Madagaskar zu. Schliesslich entspricht das Vorgehen den Verpflichtungen, welche die Schweiz anlässlich des Erdgipfels in Rio de Janeiro eingegangen ist.

Es wäre interessant, 1996 - vier Jahre nach dem Gipfel - die Auswirkungen der schweizerischen Massnahmen auf die madagassische Umwelt zu analysieren. Mit welchen Mitteln erfüllt die schweizerische Entwicklungszusammenarbeit ihre komplexe Mission, die Früchte ihres Wohlstandes mit einem Entwicklungsland wie Madagaskar zu teilen und der madagassischen Bevölkerung zu helfen, schwierige Rahmenbedingungen möglichst gut zu nutzen ?

Jeder der unten dargestellten sechs Ansätze betrachtet die Problematik und die damit verbundenen Chancen und Risiken aus einer anderen Perspektive. Die vielfältigen Ansätze könnten den Eindruck erwecken, dass die schweizerische Entwicklungszusammenarbeit verschiedene Ansätze versuchsweise « ausprobiert » und sich erst danach, je nach Ergebnis, für den einen oder anderen entscheidet. In Wirklichkeit ist darin der Wille zu sehen, die Umweltproblematik an allen Fronten anzugehen und sie zur Grundlage der Interventionen zu machen. Die folgenden Absätze behandeln die Umsetzung, die Ergebnisse und die jeweiligen Schwierigkeiten der sechs verschiedenen Ansätze.

7 Bundesrat, Leitbild Nord-Süd, Bericht des Bundesrates über die Nord-Süd-Beziehungen der Schweiz in den 90er Jahren, $1995,28 \mathrm{~S}$.

8 Direktion für Entwicklung und Zusammenarbeit (DEZA), Leitbild, 1991, 89 S. 
Der erste Ansatz bezieht sich auf die Entscheidung der DEZA, ihre forst- und landwirtschaftlichen Aktionen zu «de-sektorialisieren » und ländliche Entwicklungsprogramme zu erarbeiten, welche der « nachhaltigen Nutzung der natürlichen Ressourcen » besonderes Gewicht beimessen. Während der ersten zwanzig Jahre der Präsenz in Madagaskar betrafen die Interventionen der Schweiz verschiedene Untersektoren der pflanzenbaulichen und forstwirtschaftlichen Produktion und der Viehzucht. Im wesentlichen wurden die Institutionen in den Bereichen Berufsbildung, Ag rarforschung, Wald- und Wasserbewirtschafung technisch unterstützt. Zudem erhielt die Landbevölkerung im Rahmen von Aufforstungsprojekten, der Anlage von Trockenkulturen an den Hügeln oder der Verbesserung des Reisanbaus technische Unterstützung. Nach und nach stiess jedoch diese Form der Zusammenarbeit an bestimmte Grenzen. Bisweilen wurde neues Wissen vermittelt, ohne dass die beabsichtigten Nutzniesser die Gründe für den Bruch mit uralten Gewohnheiten verstanden. Heute kommt deshalb ein unterschiedliches Vorgehen zum Tragen, das von kulturellen, tief verwurzelten und unumgänglichen Werten ausgeht. Die lokale Bevölkerung soll aktiv mitbestimmen, ihre Bedürfnisse selbst erkennen und äussern. Die Experten der Entwicklungszusammenarbeit gelten nun nicht länger als Ausländer, die unter Missachtung der Traditionen ihr eigenes Wissen aufzwingen, sondern als echte Partner. Ausserdem tritt die Unterstützung an die Stelle des durch Geldnot und Chaos geschwächten öffentlichen Sektors, der sich immer mehr zurückzieht. Schliesslich hat die Entwicklungszusammenarbeit erkannt, dass zu gezielte und zu technische Reaktionen auf die Sorgen der Landbewohner, die sich ja auf einen gesamten Lebensraum beziehen, nicht ausreichten. Wald und Agrarland mussten unbedingt als wesentliche Bestandteile aller natürlichen Ressourcen gesehen werden, die unter dem Gesichtspunkt der Nachhaltigkeit zu nutzen sind.

Mit den Bauern in einen Dialog treten, ausgehend von kleineren Umsetzungen mit einem klaren gemeinschaftlichen Ziel (Aufforstung einer Gemeindelandparzelle, Wiederherstellung eines kleinen Bewässerungsdammes), unter Einbeziehung aller sozialen Gruppen eine Diagnose stellen, in einer Vertragsbeziehung gemeinsame Ziele und gegenseitige Verpflichtungen festschreiben : so sehen nun die üblichen Etappen im Prozess der Zusammenarbeit aus. Das Interesse der Nutzniesser steigt, aber es braucht alles mehr Zeit. Echte Beteiligung will gelernt sein; die Ergebnisse sind nicht über Nacht spürbar. Der neue Ansatz deckte zunächst zahlreiche Interessenkonflikte auf. Im Rahmen der Förderung der Nahrungsmittelproduktion auf dem Hochland beispielsweise wollten die Grundbesitzer von Reisflächen oder andern Feldern den Bauern nicht erlauben, die Kulturen selbst zu diversifizieren und daraus die ihnen zustehenden Anteile zu beziehen. Die Grundbesitzer, im übrigen häufig Stadtbewohner, stellen zwar ihr Land zur Nutzung bereit, verlangen aber im Gegenzug drei Viertel der Produktion für sich und meinen zudem, dass das restliche Viertel nicht nur eine Entlohnung, sondern sogar ein Privileg für die Landarbeiter bedeute. So sehen die Bauern zwar in der Diversifizierung eine verlockende Möglichkeit, ihre Einkommen zu erhöhen, befürchten aber, dass ihr Wunsch nach Autonomie Konflikte auslöst.

Das Engagement der Entwicklungszusammenarbeit für konstruktive Lösungen der Konflikte um die Kontrolle der natürlichen Ressourcen (und damit die wichtigsten Produktionswerkzeuge) stellt ein positives Novum dar, erfordert aber von 
den Akteuren der Entwicklung noch mehr Geduld, Takt und eine ständige Bereitschaft zur Selbstkritik. Veränderungen in der Machtstruktur können zu echter Entwicklung führen, aber auch in einem Bruch münden.

\section{$\square$ Beteiligung in der Diskussion über die Entwicklungspolitik}

Der zweite Ansatz zielt auf die Erarbeitung von Entwicklungspolitiken ab, insbesondere auf die Umsetzung des nationalen " Plan d'Action Environnemental » (PAE, siehe oben). Vor Ort leistet die Schweiz in Form von strategischer und operationeller Unterstützung der «Association Nationale des Actions Environnementales » (ANAE, Nationaler Verband für Umweltaktionen) einen Beitrag an den PAE. Ziel der ANAE ist es, den Bauernverbänden in ihren Bemühungen um eine umweltschonende Produktion zur Seite zu stehen.

Bei den Rahmenbedingungen hat die Schweiz einen wichtigen Beitrag zur Neudefinition der Forstpolitik Madagaskars geleistet und sich unter anderem verpflichtet, dessen schrittweise Umsetzung zu verfolgen. Die dabei aufgetretenen Probleme gehen weniger auf die Beziehungen der Schweiz mit den Nutzniessern als vielmehr mit den anderen Geldgebern und den nationalen Wirtschaftsakteuren zurück. Die nationalen und internationalen Experten, die im Auftrag der DEZA arbeiten, stehen den Bauern sehr nahe und bemühen sich um ein genaues Verständnis der Probleme sowie um pragmatische Vorgehensweisen. Wegen dieser engen Beziehung ist die schweizerische Zusammenarbeit besonders gut in der Lage, ihre Erfahrungen bei wichtigen Geldgebern einzubringen, welche sich nicht direkt an die Bauern richten, sondern in erster Linie die staatlichen oder nicht-staatlichen Zwischenstrukturen (namentlich in der Verwaltung der geschützten Gebiete) unterstützen. Im Beispiel der neuen Forstpolitik rechtfertigte sich die Unterstützung der Schweiz im wesentlichen durch den Realismus

der betroffenen Politik. Der Staat verfügt nicht mehr über dieselben Mittel wie während der Kolonialzeit. Die neuen Kompetenzen der Land- und Forstdirektion bestehen deshalb darin, die Prioritäten der Tätigkeitsgebiete zu bestimmen, nach Möglichkeit Aufgaben an den Privatsektor zu delegieren und die eigene zentrale Rolle im normativen Bereich zu festigen.

Auf nationaler Ebene waren die Entscheidungsträger lange hin- und hergerissen zwischen dem Wunsch nach einer ehrgeizigeren Politik - die für sie ein grösseres verfügbares Budget bedeutet hätte - und nach einer auf Nähe beruhenden Betrenung der Bauern. Schliesslich entschieden sie sich für den zweiten Weg.

Obwohl die DEZA ein viel kleineres Umweltbudget bereitstellt als die französische oder amerikanische Entwicklungszusammenarbeit, dürften ihre reichen Erfahrungen ihr erlauben, sich aktiver an der Umweltdiskussion zu beteiligen, um die Interessen der Landbevölkerung zu verteidigen.

\section{Umwelt in die öffentliche Bildung einbeziehen}

Der dritte Ansatz bezieht sich auf die Ausbildung auf der Grundstufe. Die DEZA führt ein originelles Projekt namens «Sekoly Maitso » (Grüne Schule) durch. Die Umwelt als Rahmen und Quelle des Lebens der Bauern bildet den Gegenstand eines zusätzlichen Unterrichtsfaches. Es geht darum, Kinder im Alter von 7 - 10 Jahren, die eine Schule besuchen, mit der Achtung vor der Umwelt vertraut zu machen. Keime von Obst- oder Zierbäumen zu pflanzen, sie zu pflegen, sich zu freuen, wie sie wachsen: all das bildet ein Unterrichtsfach wie Geschichte oder Rechnen. In praktischer Arbeit betreuen die Kinder eine Baum- 
schule im Schulhof. Danach verpflanzt jedes Kind sein Bäumchen in die eigene Parzelle zu Hause. Kinder, die nicht zur Schule gehen, werden zu den Schulfesten eingeladen, wo auch kleine Bäumchen verkauft werden. Mit dem Erlös kaufen Kinder entweder Schulmaterial oder führen kleinere Reparaturarbeiten an Infrastrukturen durch (zum Beispiel das beschädigte Dach eines Klassenzimmers flicken).

Zur Pilotaktion Sekoly Maitso gehören bislang nur rund fünfzig öffentliche Schulen in den ländlichen Gebieten rund um die Hauptstadt. Es ist zu hoffen, dass das sehr originelle und effiziente Projekt bald breitflächig angewandt wird. Nach der ersten Probephase (« recherche-action ») wurden im übrigen zusätzliche Kredite für die Fortsetzung der Aktion erteilt. Heute arbeitet ein kleines Team an der neuen - gelungenen - Form der Sensibilisierung der ländlichen Kinder in der Schule. Für nur vier Personen ist es sehr schwierig, die fünf Dorfgebiete im Umkreis von $50 \mathrm{~km}$ der Hauptstadt Madagaskars abzudecken. Zudem liegen die Weiler über $10 \mathrm{~km}$ im Landesinnern - dort, wo die Bewohner noch tief in ihrem Land verwurzelt sind, wo es aber auch am schwierigsten ist, mit anderen in Kontakt zu kommen und in kurzer Zeit Erfahrungen weiterzugeben. Eine Ausweitung dieses Ansatzes auf Regionen oder auf das ganze Land hat heute wegen des erheblichen Aufwandes (insbesondere an Zeit) geringe Chancen. Trotzdem bringt der Ansatz, welcher die Kinder auf dem Land so früh wie möglich einbezieht, der Landbevölkerung zwei Vorteile. Zum einen lernen die Kinder, ihre unmittelbare Umwelt zu achten, und zwar in einem Alter, in dem sie auf ihre Umgebung besonders neugierig sind. Zum anderen erhalten die Familien einen Anreiz, ihre Kinder zur Schule zu schicken, weil die dort erworbenen Erfahrungen unmittelbar im Alltag umgesetzt werden können.

Diese Sensibilisierung für die Umwelt sollte unbedingt auch in anderen Landschulen und sogar in städtischen Schulen angestrebt werden. Sekoly Maitso steht vor der grossen Herausforderung, die Zustimmung des Bildungsministeriums für die Aufnahme des Themas Grüne Schule in den Lehrplan zu gewinnen und andere Partner und Träger einzubeziehen. Für das madagassische Bildungsministerium würde das bedeuten, auf nationaler Ebene ein Lehrprogramm über Umwelt erarbeiten und kompetente Lehrkräfte ausbilden zu müssen, was zusätzliche öffentliche Investitionen erfordern und die schon seit Jahrzehnten schwerfällige Verwaltung noch stärker belasten würde. Zudem wird die Kontinuität innovativer Projekte durch die fast chronische politische Unstabilität des Landes gefährdet.

\section{$\square$ Integration der kulturellen Dimension in die ländliche Entwicklung}

Die besondere Berücksichtigung des kulturellen Erbes Madagaskars zählt zu den wichtigsten Ansätzen der schweizerischen Entwicklungszusammenarbeit. Die bei den Madagassen besonders beliebten Kunstträger zu erkennen und über diese Botschaften zur Umwelt zu übermitteln, hat sich als enormes Wagnis mit grossen Risiken und Chancen erwiesen. Erstens könnte der innovative Inhalt der Folkloretexte - das breite und wichtige Umweltthema - bei den Liebhabern der mpihira gasy auf Ablehnung stossen, weil sie an ironische Lieder gewöhnt sind, die ihnen Entspannung und gute Laune bringen. Die neue, wichtige Problematik gibt weniger Anlass zu Heiterkeit als die üblichen Themen. Kurz, lebenswichtige Anliegen werden über Träger vermittelt, die normalerweise nur der Unterhaltung dienten, was nicht auf Anhieb leicht fiel. Zweitens konnten die Zuschauer der 
mpihira gasy die dargestellten Situationen seit Jahrhunderten nachvollziehen, weil sie alle Gefühle wie Liebe, Verachtung, Begierde usw. selbst kennen. Sicherlich ist die Umweltzerstörung ein viel konkreteres und greifbareres Phänomen als die oben erwähnten traditionellen Themen ; ihre Konsequenzen zeigen sich den Bauern aber erst dann, wenn sie bewusst um sich blicken. Im allgemeinen fällt es ihnen schwer, Verantwortung für Probleme der Allgemeinheit zu übernehmen. Dies hängt mit der Mentalität der Kolonialzeit und der darauffolgenden sozialistischen Ära zusammen. Daher bestand die Gefahr, dass die Bauern sich von den Botschaften nicht richtig angesprochen fühlten und die Lieder für einen Misserfolg halten würden, die ihren eigentlichen Zweck, die Unterhaltung, verfehlten.

Die Erfahrungen der beiden letzten Jahre haben jedoch diese Befürchtungen widerlegt. Bestimmte mpihira gasy-Gruppen, die sich besonders für die Umwelt eingesetzt hatten, konnten das Interesse der Zuschauer und auch den Spass an der Vorstellung wecken. Dank dem Gleichgewicht zwischen deutlichen Warnungen vor der Umweltzerstörung und beschwingten Rhythmen ist es ihnen gelungen, skeptische Zuhörer zu überzeugen.

Um mit ihrer Botschaft auch das städtische Publikum zu erreichen, das sich in der Regel weniger für die mpihira gasy begeistert als die Landbevölkerung, wandte sich die DEZA im Rahmen der Kampagne für kulturellen Austausch « Gasikara Maitso Volo » (wörtl. Madagaskar, grüne Landschaften) an schweizerische und madagassische Variété-Sänger. Diese Künstler traten in Konzerten in grösseren Schweizer Städten und in Madagaskar als « Boten » auf. Einige Lieder wurden zu echten « Hits ». Dank Gasikara Maitso Volo konnte die Grundlage für eine vielversprechende Zusammenarbeit unter ökologisch motivierten Sängern und Medien gelegt werden. Allerdings sind die erwünschten Auswirkungen des kulturellen Weges trotz des Durchbruchs vor Ort ausgeblieben. Das Problem bestand darin, die neue Orientierung der Künstler zu fördern, damit ihr Engagement nicht nachliess, und vom öffentlichen Sektor eine kontinuierliche Unterstützung zu erhalten. Trotz des weitgehend positiven Echos in den schweizerischen und madagassischen Medien war der Kampagne kein Erfolg beschieden. Zum einen äusserten bestimmte Kritiker, die nur die musikalische Seite der Kampagne bewerteten, Zweifel an Sinn und Zweck und an den Kosten. Dadurch wurde das Engagement der öffentlichen Behörden in einer schon angespannten Finanzlage geschwächt. Zum anderen fiel die Entscheidung über die Fortsetzung der Kampagne in eine Zeit der Regierungswechsel in Madagaskar; vier verschiedenen Kulturministern innerhalb von sieben Monaten ist es nicht gelungen, einen neuartigen, aber nicht leicht umsetzbaren Ansatz zu fördern. Die Verantwortlichen stehen unter Leistungsdruck, sie fühlen sich bisweilen « von der Zeit überrannt » und geben auf, wenn die Resultate auf sich warten lassen. Gerade im «empfindlichen» Bereich jedoch braucht die Entwicklungszusammenarbeit Zeit... Wer hätte allen Ernstes glauben können, dass es nach nur einer Konzertsaison keine Buschbrände mehr geben würde?

$\square$ Öffentlichkeit und Entscheidungsträger über die Umweltanliegen informieren Eines der heikelsten Themen im Umgang mit den madagassischen Bauern war der Gebrauch von Pestiziden. Seit in den früheren 70er Jahren die « DDT-Kultur » den jahrhundertealten natürlichen Praktiken ein Ende bereitet hatte, setzten die Landwirte in ihren Betrieben Chemieprodukte auf der Basis von DDT in 
hohen Mengen ein. Auch alle anderen wegen ihrer Toxizität in den Industrieländern verbotenen Pestizide, die dann im Rahmen der bilateralen Hilfe gratis über Händler und gemeinschaftlich organisierte Chemikalien- und Pharmazeutikalager abgegeben wurden, nahmen die Bauern gern entgegen. Der jüngste Umweltansatz der DEZA besteht deshalb darin, die Benutzer von Pestiziden und die Behörden über die Risiken chemischer Substanzen für den Menschen und für die Umwelt aufzuklären.

Gestützt auf ihre Erfahrungen während zwölf Jahren in Sachen Pestiziden sieht die DEZA mit dem Projekt Voarisoa (gute Schöpfung) einen sehr unkonventionellen Ansatz vor: Die einen sollen vor Chemikalienmissbrauch gewarnt, die anderen für «good practices » (Gesetze, Schulung usw.) im Bereich der gefährlichen Güter sensibilisiert werden. Voarisoa bringt die Nutzniesser des Projekts in direkten Kontakt mit den Entscheidungsträgern und dient so lediglich als Forum, wo sich potentielle Partner ausserhalb der formellen Kreise treffen können. Im übrigen ist Voarisoa mit nur einem ständigen Experten und einem Sekretariat von zwei Mitarbeitern ausgestattet; eine Reihe nationaler interessierter Berater können bei Bedarf herangezogen werden. So war es im Rahmen von Voarisoa zum Beispiel möglich, dass Spezialisten für Agrarpestizide die Kurse der Beauftragten des Gesundheitsministeriums über die hausinterne Bekämpfung der Malariamücke unterstützten und verbesserten. Ferner erhielten Gemüseproduzenten Zugang zu den jüngsten « Funden » der Forscher, damit sie ihre Kulturen nach ökologischen und preiswerten Methoden anbauen können. Auf Verwaltungsebene wurden die Beauftragten des Pflanzenschutzamtes in ihren Bemühungen um regelmässige Qualitätskontrollen der auf dem Markt angebotenen Pestizide unterstützt. Schliesslich wurde der madagassische Ausschuss für die Zulassung chemischer Produkte über die jüngsten einschlägigen Fortschritte insbesondere der FAO und über die relevanten internationalen Konventionen informiert.

Das Lern- und Informationsnetz findet bei den Produzentenverbänden und den Pestizidhändlern ein reges Interesse, zumal sie sich nicht formell an das Projekt gebunden fühlen und nicht befürchten müssen, in ein administratives Verfahren hineingezogen zu werden. So reagieren sie positiv auf Besuche des VoarisoaTeams und beantragen sogar selbst Informationssitzungen. Die « Erbringer » der Dienstleistungen von Voarisoa ihrerseits freuen sich, dass der Informationsbedarf der Öffentlichkeit ohne grossen Aufwand und kostspielige Aktionen gedeckt werden kann.

Selbst in dieser vielversprechenden Atmosphäre stösst man jedoch bisweilen auf grosse Hindernisse : den Widerstand mancher skrupelloser (Weiter)Verkäufer gegen die Kontrolle ihrer Waren, das Fehlen eindeutiger Gesetze über Impaktstudien bei der Ansiedlung neuer Industrien und vor allem die Schwäche des öffentlichen Sektors bei der Überprüfung der Einhaltung der geltenden Bestimmungen. Erschwerend kommt hinzu, dass die Importe und der Absatz von Pestiziden über mehrere Kanäle erfolgen : Niederlassungen ausländischer Chemiefirmen, die einzig an jährliche Einfuhrkontingente gebunden sind; «Freischützen », die auf Konjunkturgewinne spekulieren; freier Handel mit unkontrollierten chemischen Inputs ; und Missbräuche profitgieriger Politiker. Nicht zuletzt verweigern lokale Firmen, die in der Neuverpackung von Chemieprodukten tätig sind, jegliche Auskunft über ihre Reinigungs- und Abflussysteme. Das erschwert den Informationsaustausch und ist im übrigen ein Beweis dafür, wie sehr diese Art von Zusammenarbeit bisweilen « stört ». 
Trotzdem werden derzeit Möglichkeiten für eine bessere Kommunikation zwischen Importeuren/Verkäufern und Benutzern von Pestiziden ausgelotet. In der Stadt spielt die Presse bei der Information der breiten Öffentlichkeit eine Schlüsselrolle. Auf dem Land bilden die Bauernverbände echte Quellen des Erfahrungsaustauschs und der Erkenntnisse zu den neuen umweltverträglicheren Landwirtschaftspraktiken.

\section{$\square$ Die Umweltauswirkungen des Programms Madagaskar-Schweiz systematisch auswerten}

Mit den verschiedenen oben geschilderten Ansätzen verfügt die madagassischschweizerische Entwicklungszusammenarbeit für ihre Aktionen über eine Fülle von Lernquellen und Werkzeugen mit einer klaren umweltbezogenen Ausrichtung. Abgesehen von den unterschiedlichen Ansätzen und ihrem potentiellen Anwendungsgebiet steht die Frage der Umweltauswirkungen von Aktionen mit anderer Ausrichtung (ländliche Infrastrukturen, Gesundheit, usw.) noch offen. Wie kann man gewährleisten, dass alle Aktionen des Programmes ihre Umwelt und ihren Einfluss darauf berücksichtigen?

Um eine Teilantwort auf diese Frage zu erhalten, hat die DEZA das Geographische Institut der Universität Bern (Gruppe für Entwicklung und Umwelt) beauftragt, ein Raster mit effizienten und sachdienlichen Indikatoren zu erarbeiten. Anhand dieses Instruments wurde 1995 eine erste Aktion, ein Strassenunterhaltsprojekt im grössten Reisanbaugebiet Madagaskars, analysiert. Dank diesem Projekt gelang es den Bewohnern der Region, Erosion und Abholzung einzudämmen, das Strassendränagesystem zu verbessern, Erdrutsche an den unteren Hängen zu begrenzen, die regelmässige Wartung der Strasse selbst zu übernehmen und bei allen Benutzern die Einhaltung der Vorschriften durchzusetzen.

In einem Test-Workshop ${ }^{9}$ bemühten sich Vertreter des Projektteams, Entscheidungsträger, Wirtschaftsakteure, Berater und auswärtige Personen, das Verhalten der gesellschaftlichen Gruppen, für welche die fragliche Strassenachse wichtig war, besser zu verstehen (Bewohner der Dörfer, Anrainer, Transporteure usw.). In der Diskussion befasste man sich mit den Beziehungen der sozialen Gruppen zu den Projektkomponenten und mit den Auswirkungen der auf unterschiedlichen Ebenen durchgeführten Aktionen. Dank diesem Austausch konnten mögliche Konfliktszenarien zwischen Gruppen von Akteuren und/oder Nutzniessern erkannt werden. Diese wurden aufgefordert, sich mit solchen Situationen auseinanderzusetzen, ohne ihnen auszuweichen. Der Versuch, eine Aktion nicht getrennt zu betrachten, sondern auch die breiteren Auswirkungen berücksichtigen, bietet zahlreiche Vorteile, insbesondere was die Sensibilisierung der verschiedenen Akteure angeht. Positiv ist an dieser Vorgehensweise ferner, dass die hauptsächlichen Interessenkonflikte aufgezeigt werden. Das bedeutet zwar noch keine Lösung, aber ein möglichst objektives Problembewusstsein der wichtigsten Akteure stellt schon einen Schritt in die richtige Richtung dar.

Unser Beispiel - Unterhalt einer zentralen Strassenachse - soll veranschaulichen, wie solche Konflikte aussehen können. Den Bauern bringt die besser zugängliche Strasse Vorteile ; der Verkehr nimmt zu, es werden mehr Waren transportiert, die Preise sinken. Die Bauern bieten ihre Ware am Strassenrand feil. Sie bringen

9 DEZA Compte-rendu de l'atelier « Routes et Environnement », 1995, unpubl., 38 S. 
gemeinsam Regenbarrieren an, um die Strasse in einem guten Zustand zu erhalten. Andererseits leiden die einflussreichen Transporteure sowie die Reis- und Holzkohlenverkäufer darunter, dass sie nicht länger den schlechten Strassenzustand vorschützten können, um mit den Grundnahrungsmitteln zu spekulieren. Sie werden daher alles daran setzen, um die Projektverantwortlichen und die durchführenden Stellen von ihrem Vorhaben abzubringen. Im vergangenen Juli wurde der wichtigste Schweizer Sachverständige für dieses Programm umgebracht. Wir können nur hoffen, dass dieses traurige Ereignis nichts mit dem Einsatz der DEZA zu tun hat. Die Untersuchungsergebnisse stehen noch aus, und die Verantwortlichen machen sich weiterhin Sorgen.

Kehren wir zum eigentlichen Thema zurück. Der gewählte Ansatz ermöglichte ein besseres Verständnis des Aktionsrahmens und der komplexen Verbindungen der Aktion mit ihrem Umfeld. Die DEZA hat beschlossen, diesen Ansatz auf systematischer Basis fortzusetzen. Bei jeder Evaluations-, Orientierungs- und Planungsetappe werden die Angestellten der DEZA eine Analyse durchführen und die Aktion entsprechend korrigieren und ändern. Ferner werden Indikatoren bestimmt, anhand deren Entwicklung zu gegebener Zeit die geeigneten Massnahmen zu treffen sind.

Der systematische Folge- und Evaluationsprozess der Umweltauswirkungen erfordert viel Zeit und Engagement. Es ist nicht immer leicht, die richtigen Personen zum richtigen Zeitpunkt zu mobilisieren. Die verschiedenen betroffenen Akteure auf die Umweltproblematik aufmerksam zu machen und sie zu bewegen, sich prioritär um spezifische Lösungen zu bemühen, ist eine schwierige Aufgabe. Zudem müsste bei einer Ausdehnung solcher Impaktanalysen auf das gesamte Zusammenarbeitsprogramm zwischen Madagaskar und der Schweiz, dem unterschiedlichen Rahmen und dem jeweiligen Reifegrad der Aktionen Rechnung getragen werden. Da es sich um spezifische Aktionen, Teilnehmer und auch Interessen handelt, ist noch nicht abzusehen, welche Reaktionen dann für bestimmte Problematiken vorbereitet werden müssen. Auch hier gilt, dass die Evaluationsinstrumente ihre Wirkungen erst nach einer gewissen Zeit entfalten.

\section{GESAMTBILANZ}

Welche Bilanz lässt sich aus dieser Vielfalt an Ansätzen im Dienst der Entwicklung ziehen ? Wie bewertet die DEZA die Gesamtergebnisse des Entwicklungsprogrammes in Madagaskar?

Die Programmverantwortlichen sind überzeugt, dass der Ansatz der DEZA in Madagaskar die Grundsätze der nachhaltigen Entwicklung erfolgreich umgesetzt hat. Mit ihren Aktionen ermutigt die schweizerische Entwicklungszusammenarbeit die Landbevölkerung, der nächsten Generation ein nachhaltig bewirtschaftetes und vor Übernutzung geschütztes Erbe weiterzugeben. Die schweizerische Entwicklungszusammenarbeit sieht in ihrem Engagement konkrete Erfolge, bisweilen überschattet von negativen Aspekten, welche sie jedoch in Herausforderungen umwandeln möchte. Hier eine kurze Zusammenstellung.

Zunächst veranlasste der von der DEZA vertretene Beteiligungs-Ansatz die Berater und die Bauern, sich spontan zusammenzuschliessen und die Probleme in der Landwirtschaft mit vereinten Kräften zu lösen. Je mehr die Berater auf die 
Bauern zugehen und ihre Traditionen respektieren, desto grössere Sympathie wird ihnen entgegengebracht. Solche Resultate verstärken zudem bei den Bauern das Bewusstsein ihrer Eigenverantwortung.

Im übrigen führte die Aufnahme des Umweltkonzeptes in die Aktionen zu einer engeren und intensiveren Zusammenarbeit zwischen den Beratern und der lokalen Bevölkerung. Die ersten erhalten die Gelegenheit, unterschiedliche Ansätze zugunsten der Umwelt umzusetzen, die zweiten werden dank der technischen, methodologischen und strukturellen Unterstützung besser mit Umweltfragen fertig und können ihre natürlichen Ressourcen nachhaltiger bewirtschaften.

Schliesslich versucht die DEZA, in einem Land mit mehrheitlich sehr junger Bevölkerung gerade die Kinder ab dem ersten Schuljahr einzubeziehen ; so können sich umweltgerechte Verhaltensweisen in der Bevölkerung von Generation zu Generation immer fester verankern.

Diese drei noch unvollständigen Ergebnisse bilden die notwendige Ausgangslage für eine nachhaltige Entwicklung. Die junge Generation und die Bauern müssen gemeinsam die erforderliche Energie aufbringen, um die Entscheidungsträger zu einer ökologischen Nutzung von Boden, Meer und Wald zu bewegen. Bis solch wichtige Errungenschaften wirklich Fuss fassen, hat die madagassisch-schweizerische Zusammenarbeit noch zahlreiche strukturelle, politische und strategische Hindernisse zu überwinden.

Damit sich das Umweltbewusstsein überhaupt erst entwickeln kann, müssen die Behörden klare Strategien zur Nutzung der natürlichen Ressourcen des Landes ausarbeiten. Für ein Land mit einem BSP von knapp über 200 US\$ pro Kopf ist es oft schwierig, eine umweltfreundliche Politik zu betreiben und gleichzeitig zwar flächen- und inputintensive Exportkulturen, die aber Devisen und kurzfristige Gewinne bringen, zu fördern. Die Herausforderung liegt im Interessenkonflikt zwischen Bauern einerseits, welche in den Tag hinein (über)leben müssen, aber fähig sind, Umweltaktionen engagiert durchzuführen, und den öffentlichen Behörden auf jeder Ebene, welche hin- und hergerissen sind zwischen einer überintensiven Exporttätigkeit zur Ausgleichung ihrer chronisch defizitären Handelsbilanz und einer Normalisierung der Boden-, Wald- und Meeresnutzung dank der Finanzhilfe der multilateralen Zusammenarbeit. Die Rolle der internationalen Zusammenarbeit besteht darin, einen Reflexionsprozess über die Reglementierung anzuregen, welcher die öffentlichen Behörden aller Hierarchiestufen veranlassen soll, Gesetze zur Achtung der Umwelt einzuführen.

Eine zweite Schwierigkeit bezieht sich auf die Grundbesitzverhältnisse. Wenn die Bauern ihre Felder besitzen und nicht nur benutzen könnten, so würden sie dem Schutz ihrer Existenzgrundlagen sicherlich mehr Zeit und Energie widmen. Hier wird jedoch ein Komplex von umfassenden Privilegien berührt ; erhebliche Fortschritte sind noch erforderlich, um den Grundbesitz für alle zu gewährleisten. Im Fall Madagaskars hat sich eine breite, wenngleich noch schüchterne Bewegung gebildet, die darauf hinarbeitet und die es verdiente, von den NROs, den Entwicklungsorganisationen und vom privaten Sektor nach Möglichkeit unterstützt zu werden. Es bleibt zu hoffen, dass die Grundbesitzverhältnisse in Madagaskar deutlich verbessert werden können, bevor die Spirale Armut Umweltzerstörung in sozialen Spannungen eskaliert.

Im sich anbahnenden Dialog unter den Nationen zur Erhaltung der bedrohten Ökosysteme haben alle daraus zu gewinnen, die Erfahrungen regelmässig in 
internationalen Foren auszutauschen, auf ihre Nachbarn zu hören und die Bilanz der Erfahrungen - auch im Bereich der Entwicklungszusammenarbeit - vorzulegen, um die Dynamik für eine neue Um-Weltordnung aufrechtzuerhalten. Eine Utopie?

\section{Glossar der madagassischen Begriffe}

\begin{tabular}{|c|c|}
\hline Gasikara Maits & $\begin{array}{l}\text { Vom madagassichen Kulturministerium im Mai } 1995 \text { geprägter Slogan } \\
\text { zur Bezeichnung des Sensibilisierungsprojekts im Bereich Kultur und } \\
\text { Umwelt, welches das Ministerium und die schweizerische Entwick- } \\
\text { lungszusammenarbeit gemeinsam umsetzten. Der Slogan erinnert an } \\
\text { die grünen Landschaften Madagaskars. }\end{array}$ \\
\hline kabary : & $\begin{array}{l}\text { bedeutet « Rede », bezeichnet aber in Madagaskar die Wortgefechte } \\
\text { und rhetorischen Inszenierungen. }\end{array}$ \\
\hline lavaka: & $\begin{array}{l}\text { geologischer Fachbegriff; bezieht sich auf erosionsbedingte Phä- } \\
\text { nomene (Erdeinbrüche, die sich in spektakulären Klüften und Rissen } \\
\text { an den Hügelhängen äussern). }\end{array}$ \\
\hline Lova Soa : & $\begin{array}{l}\text { wörtlich übersetzt « wertvolles Erbe »; bezieht sich auf ein Projekt zur } \\
\text { Bewirtschaftung der durch Buschbrand und Erosion beschädigten } \\
\text { Hügel des madagassischen Hochlands. }\end{array}$ \\
\hline mpihira-gasy: & Folkloresänger und -tänzer. \\
\hline monka: & $\begin{array}{l}\text { bezeichnet brachliegendes Land, auf dem sich eine Sekundärvegeta- } \\
\text { tion (nach Kahlschlag und Buschbränden) gebildet hat. }\end{array}$ \\
\hline Sekoly Maitso : & Name eines Schweizer Projektes; « grüne » oder ökologische Schule \\
\hline tanety: & bedeutet Hügel. \\
\hline Voarisoa : & wörtlich übersetzt « gute Schöpfung ». \\
\hline
\end{tabular}

